\title{
«Une constellation dans la nuit de l'histoire, une tache lumineuse devant les ténèbres de la mort »: l'œuvre d'Ernst Wilhelm Nay vue par Werner Haftmann
}

"A constellation in the night of history, a bright spot in front of the darkness of death » : the work of Ernst Wilhelm Nay as seen by Werner Haftmann

\section{Vincenza Benedettino}

\section{OpenEdition}

Journals

Édition électronique

URL : https://journals.openedition.org/cel/15760

DOI : $10.4000 / c e l .15760$

ISSN : 2262-208X

Éditeur

École du Louvre

\section{Référence électronique}

Vincenza Benedettino, « « Une constellation dans la nuit de l'histoire, une tache lumineuse devant les ténèbres de la mort » : I'œuvre d'Ernst Wilhelm Nay vue par Werner Haftmann », Les Cahiers de l'École du Louvre [En ligne], 16 | 2021, mis en ligne le 04 juin 2021, consulté le 30 juillet 2021. URL : http:// journals.openedition.org/cel/15760; DOI : https://doi.org/10.4000/cel.15760

Ce document a été généré automatiquement le 30 juillet 2021.

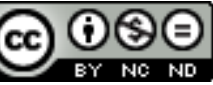

Les Cahiers de l'École du Louvre sont mis à disposition selon les termes de la licence Creative Commons Attribution - Pas d'Utilisation Commerciale - Pas de Modification 4.0 International. 
«Une constellation dans la nuit de l'histoire, une tache lumineuse devant les ténèbres de la mort » : l'œuvre d'Ernst Wilhelm Nay vue par Werner Haftmann

"A constellation in the night of history, a bright spot in front of the darkness of death » : the work of Ernst Wilhelm Nay as seen by Werner Haftmann

Vincenza Benedettino

1 La relation professionnelle et amicale entre l'historien de l'art Werner Haftmann ${ }^{1}$ (1912-1999) et l'artiste Ernst Wilhelm $\mathrm{Nay}^{2}$ (1902-1968) présente l'une des expériences les plus significatives en termes de construction historiographique de l'art dans la République Fédérale d'Allemagne de l'après-guerre ${ }^{3}$. Cet article propose de dégager les étapes fondamentales d'un itinéraire que nous pouvons tout à fait considérer comme un véritable processus de consécration de l'œuvre de Nay dans l'histoire de l'art entrepris par Haftmann ${ }^{4}$. Si par son travail critique Haftmann a contribué de façon déterminante au succès artistique de Nay, en retour son œuvre a constitué l'exemple par excellence du soutien à la théorie du triomphe de l'abstraction, postulée par Haftmann dans le contexte historique et politique de la République Fédérale d'Allemagne dès 1946. À travers l'analyse de la correspondance entre l'historien de l'art et l'artiste, lesquels étaient liés d'amitié dès avant la Seconde Guerre mondiale ${ }^{5}$, ainsi que d'une sélection représentative d'ouvrages et de catalogues d'expositions écrits par Haftmann, nous démontrerons les stratégies de cette interaction réciproque, afin d'apporter un regard nouveau sur les processus de reconnaissance et de consécration des œuvres et des théories critiques en histoire de l'art. Bien que le rapport étroit entre Haftmann et Nay, tout comme l'influence du travail critique de l'historien de l'art sur la réussite de la peinture de l'artiste, soient des faits reconnus, aucune étude monographique n'a jusqu'à présent été dédiée à ce sujet ${ }^{6}$. À partir d'une présentation 
chronologique des écrits et de la correspondance, nous contextualiserons la place attribuée par Haftmann à la peinture de Nay dans son analyse du développement de l'histoire de l'art du $\mathrm{XX}^{\mathrm{e}}$ siècle. Tout en mettant en lumière les thématiques distinctives de l'œuvre de Nay concernant la couleur et la forme, ainsi que le rapport avec la figuration et la représentation du réel, Haftmann a contribué à édifier ce parcours artistique en exemple d'un style moderne et indépendant, par opposition aux courants se développant dès la fin des années 1950 en Europe et aux États-Unis.

\section{E. W. Nay dans l'encyclopédie Malerei im 20. Jahrhundert}

2 La canonisation de Nay en tant qu'artiste fondamental dans l'histoire de l'art contemporain allemand eu officiellement lieu lors de la parution de l'encyclopédie de Haftmann, Malerei im 20. Jahrhundert, en 19547. Dans la cinquième partie de cet ouvrage, Malerei der Gegenwart. Kunst seit 1945, Haftmann revendique en effet l'importance de la peinture abstraite de Nay: l'artiste, réputé comme « un des plus importants talents à disposition de la peinture aujourd'hui ${ }^{8}$ ", c'est-à-dire en 1950-1951, aurait enfin apporté "la première solution approfondie ${ }^{9}$ » au problème de l'autonomie de la couleur, en lui donnant une valeur propre, et non plus juste un rôle subordonné d'accompagnement de la forme ${ }^{10}$.

3 Haftmann retrace ensuite le développement de l'art de Nay en expliquant les étapes qui ont marqué l'avancement de sa peinture, et en suivant l'énoncé de l'artiste selon lequel «faire de la peinture, c'est former l'image à partir de la couleur ${ }^{11}$ ». Haftmann interprète l'ensemble de la production artistique de Nay à l'aune de cette déclaration, comme un processus dès l'origine tourné vers la recherche de l'autonomie de la couleur sur la forme. Le parcours de l'artiste, inspiré au début de son activité par les expressionnistes Edvard Munch, Emil Nolde et Ernst Ludwig Kirchner, aurait donné, selon Haftmann, des premiers résultats significatifs avec les œuvres dites Lofoten de 1936, peintes en Norvège pendant un séjour aux Îles Lofoten et reproduisant des sujets figuratifs. Ces dernières furent suivies par les tableaux créés en 1943 pendant son service militaire en France, caractérisés par un changement fondamental qui voit la couleur acquérir de plus en plus d'importance en dépit de la figuration. Dans les œuvres datées de 1946, Haftmann constate que la couleur "se détache ${ }^{12}$ » encore plus de la représentation du réel et que cette production artistique anticipe le passage suivant entrepris par l'artiste. Dans les tableaux des années 1950 et 1951, la surface de la toile célébrait une pure manifestation de la couleur, ne donnant plus lieu qu'à des associations de type musical. Il n'y avait désormais plus aucune trace de référence au monde de la figuration, les titres mêmes renvoyant exclusivement aux couleurs, véritables protagonistes de cette peinture. Enfin, à partir de 1956, les éléments formels et rythmiques furent réduits à un motif élémentaire, marquant la période dite des "disques », dans laquelle la couleur n'était plus porteuse de significations autres mais une configuration exclusivement en soi. Dans ces œuvres, selon Haftmann, « la couleur seule soutient l'image ${ }^{13} »$. 


\section{5-1964 : la promotion de l'art abstrait comme art de la modernité en RFA}

En 1955, Arnold Bode, commissaire de la documenta à Kassel, fait appel à Haftmann pour cette exposition temporaire importante qui, lors de sa troisième édition, fut aussi surnommée le « musée des 100 jours $^{14}$ ». Haftmann devint responsable de la conception théorique des trois premières éditions, pendant lesquelles l'œuvre de Nay fut toujours exposée. La documenta de 1955, intitulée Kunst des XX. Jahrhundert. Internationale Ausstellung ${ }^{15}$ fut conçue comme une présentation exhaustive des courants et des artistes majeurs du XX $\mathrm{X}^{\mathrm{e}}$ siècle $^{16}$. À côté de pionniers de l'art moderne comme Pablo Picasso, Paul Klee, Vassily Kandinsky, Henri Matisse, furent accrochées les œuvres de Nay, Fritz Winter, Hans Hartung, Wols, afin de souligner la continuité entre l'art abstrait contemporain et les expériences révolutionnaires de déconstruction de l'image du début du XX siècle $^{17}$.

5 En 1959, la deuxième documenta, intitulée Kunst nach 1945. Internationale Ausstellung, fut dédiée à l'art après $1945^{18}$. À l'occasion de son discours d'inauguration, Haftmann expliqua qu'après les bouleversements de la période nazie et du conflit mondial, la peinture contemporaine s'exprimait désormais au moyen d'un langage artistique abstrait n'ayant plus pour but la reproduction de la nature et de la réalité ${ }^{19}$. La salle principale de l'exposition, située au Museum Fridericianum, était dominée par Das Freiburger Bild de Nay (fig. 1), réalisé en 1956 pour l'Institut de Chimie de l'Université de Fribourg ${ }^{20}$. Le tableau, mesurant $255 \times 655 \mathrm{~cm}$, était placée juste en face de la peinture de 1946, Being with (Être Avec) de Roberto Matta, également de dimensions monumentales $(221 \times 457,2 \mathrm{~cm})$ et avec laquelle elle se confrontait directement ${ }^{21}$. Selon l'historien de l'art Harald Kimpel, le visiteur de cette salle se trouvait plongé dans une "jungle» de toiles, regroupant un grand nombre d'œuvres, entre autres de Pierre Soulages, Hans Hartung, Roberto Matta, Jean-Paul Riopelle, Alberto Burri et Fritz Winter, n'ayant qu'une seule chose en commun, le fait de ne pas être figuratives ${ }^{22}$. 
Figure 1.

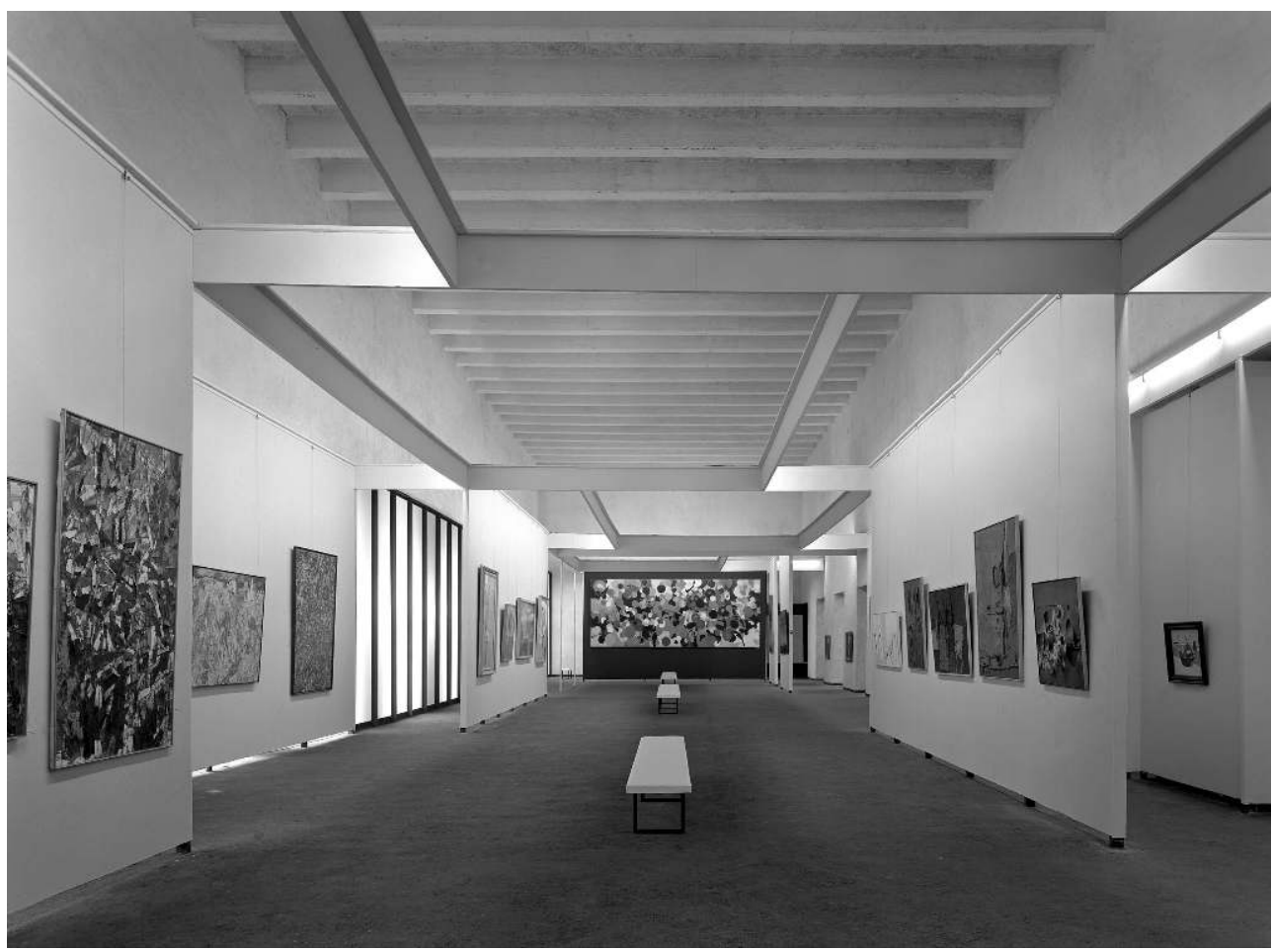

documenta II, 1959, Kassel. Salle principale du Museum Fridericianum. Au fond au centre est accroché le tableau d' E. W. Nay Das Freiburger Bild, 1956, huile sur toile, 255 × 655 cm, Freiburg im Breisgau, Land Baden-Württemberg, Chemisches Institut der Universität Freiburg.

(C) Bildarchiv Foto Marburg/documenta archiv/Günther Becker

6 Enfin, en 1964, la troisième documenta, intitulée Internationale Ausstellung, visait à mettre en lumière la portée des développements les plus récents dans le domaine de l'art abstrait. Cependant, l'apport des maîtres de la première moitié du $\mathrm{XX}^{\mathrm{e}}$ siècle $\mathrm{y}$ était à nouveau thématisé ${ }^{23}$. Haftmann expliqua cette fois, toujours à l'occasion de son discours d'ouverture, que l'individualité et la personnalité unique de chacun des artistes présentés étaient au cœur de sa conception de l'exposition ${ }^{24}$. C'est à cette occasion également qu'il affirma: "l'art est ce que les artistes connus font " déclaration qui provoqua de vives controverses ${ }^{25}$. Arnold Bode, grâce à la médiation de Haftmann, avait réussi à convaincre Nay, qui craignait de faire tomber son œuvre dans le domaine de la décoration, de réaliser trois toiles de grandes dimensions pour les accrocher au plafond ${ }^{26}$. Avec Drei Bilder im Raum, réalisées par Nay en 1964 (fig. 2), Bode contribua à mettre en scène cette œuvre d'une manière qui suscita à la fois beaucoup d'admiration et de critiques ${ }^{27}$. Ces accrochages, avec la présentation de trois toiles de Sam Francis créés en 1956-1957 pour la Kunsthalle de Bâle en forme d'hexagone, et des Absurdes Berliner Tagebuch (Plurimi di Berlino) d'Emilio Vedova, constituèrent les points forts de l'exposition, non seulement du point de vue de la mise en scène mais aussi parce qu'ils représentaient la célébration des courants non figuratifs ${ }^{28}$. Encore une fois, la place attribuée à l'œuvre de Nay fut symbolique de l'importance que son art joue dans la conception historique et artistique de Haftmann. 
Figure 2.

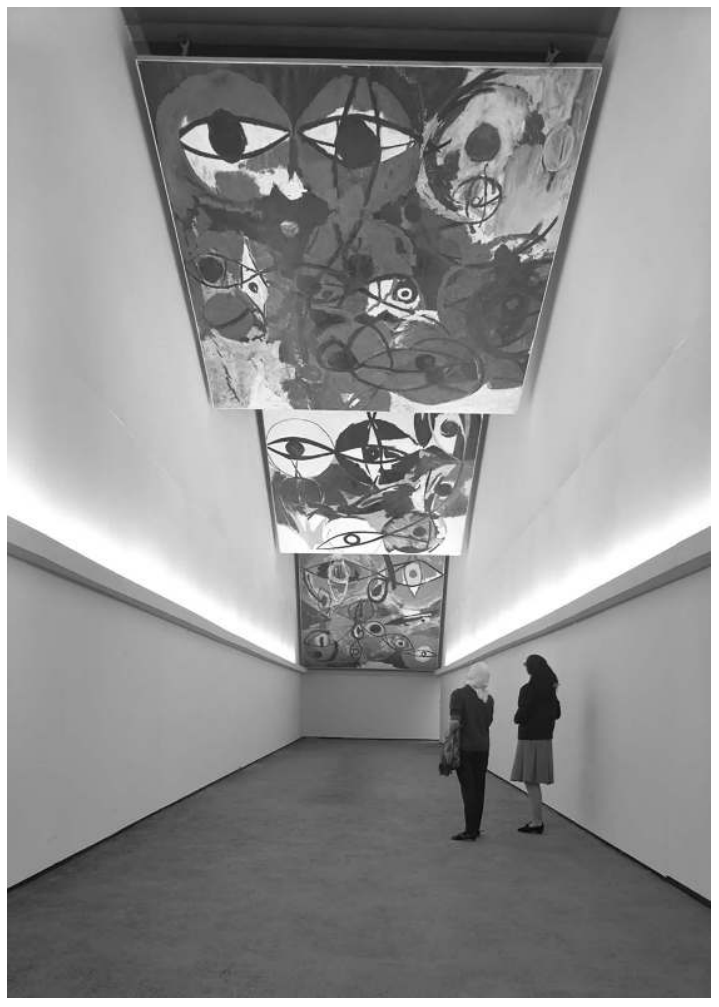

documenta III, 1964, Kassel. E. W. Nay, Drei Bilder im Raum : Documenta Bild A (Rot-Grün) ; Documenta Bild B (Blau-Rot-Gelb) ; Documenta Bild C (Weiß-Schwarz-Grau), 1964, huile sur toile, chacun 396 × 396 cm, collection privé, en prêt au Bundeskanzleramt, Berlin.

\section{7 : la présentation de l'art abstrait comme « nouvel art allemand » aux USA}

Haftmann, grand « apologiste de l'art informel ${ }^{29}$ », «pape de l'art abstrait ${ }^{30}$ », ainsi qu'il était alors appelé en Allemagne non sans une connotation polémique ${ }^{31}$, fut nommé en 1957 commissaire de la section dédiée à la peinture dans le cadre de la grande exposition German art of the twentieth century, organisée par Andrew Carnduff Ritchie, directeur du département de peinture et sculpture du MoMA à New York ${ }^{32}$.

Cette exposition, organisée sous l'égide du gouvernement de la République Fédérale d'Allemagne, se présentait comme la plus complète sélection d'art allemand réunie aux États-Unis après l'accrochage d'Alfred H. Barr Jr. au MoMA en 1931. Elle comprenait une sélection de 175 tableaux, sculptures et dessins et visait à montrer un panorama représentatif de la production artistique allemande ${ }^{33}$. Comme Ritchie l'explique dans l'introduction du catalogue, l'exposition s'ouvre avec les courants de l'expressionnisme Die Brücke et Der blaue Reiter, se poursuivant avec la Nouvelle objectivité et les artistes du Bauhaus, et se terminant par une sélection d'artistes contemporains de premier plan ${ }^{34}$.

Dans la section consacrée à la peinture contemporaine de l'après-guerre, l'exposition fut concentrée par Haftmann sur l'œuvre de trois artistes abstraits : E. W. Nay (fig. 3), Fritz Winter (1905-1976) et Theodor Werner (1886-1969). Dans une lettre du 22 mars 1957 adressée au directeur de l'Académie des Beaux-Arts de Karlsruhe, Kurt Martin ${ }^{35}$, à 
laquelle Haftmann ajoute son texte pour la préface du catalogue de l'exposition, l'historien de l'art affirme avoir choisi de focaliser la conception de l'accrochage sur ces artistes, qu'il considère comme «le trépied» de la peinture allemande contemporaine ${ }^{36}$.

Figure 3.

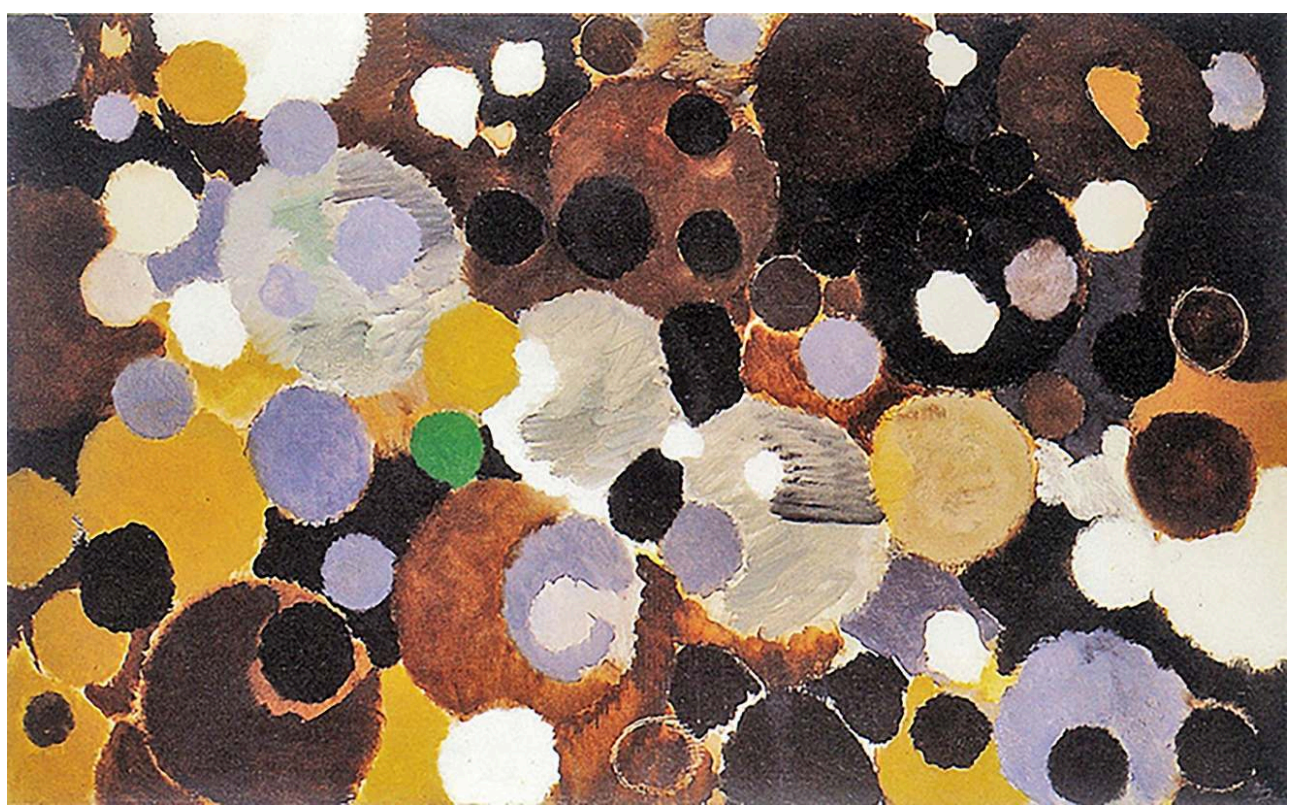

E. W. Nay, Dunkle Melodie, 1956, huile sur toile, 125,5 × $200 \mathrm{~cm}$, nº d' inventaire 3.9 (Legat Heinrich Gebert), Kunstmuseum Appenzell.

(c) Heinrich Gebert Kulturstiftung/Kunstmuseum Appenzell

Dans un paragraphe de la préface, intitulé Painting Today, il explique en effet que pendant l'après-guerre en Allemagne, ainsi que dans le reste d'Europe, l'art abstrait est devenu le style artistique prédominant. Il développe l'idée selon laquelle, puisqu'en Allemagne de l'Ouest il n'y a eu aucune influence politique en faveur du réalisme socialiste, l'art figuratif, sans disparaittre complètement, ne consiste plus qu'en un " lyrisme calme, discret, souvent mélancolique et plein de virtuosité », tandis que les peintres Wassily Kandinsky, Piet Mondrian et Paul Klee sont tout à fait considérés comme les artistes les plus influents ${ }^{37}$. Haftmann met également en lumière la manière dont l'œuvre de Willi Baumeister (1889-1955), artiste déjà bien établi avant la guerre, a joué un rôle décisif pour la diffusion de l'abstraction auprès d'une génération de peintres plus jeunes, dans les premières années de l'après-guerre. Cette influence a mené au développement d'une part, d'une peinture comme celle de Werner Gilles (1894-1961) et Heinz Trökes (1913-1997), qui dans sa structure abstraite préserve encore des éléments figuratifs; d'autre part, d'une peinture concrète qui s'est développée autour de Max Bill (1908-1994) et Friedrich Vordemberge-Gildewart (1899-1962) à la Hochschule für Gestaltung de Ulm. Entre ces deux pôles, Haftmann situe une orientation artistique "méditative ${ }^{38}$ ", renvoyant au " pouvoir poétique de la forme abstraite » et représentée par l'œuvre de Conrad Westphal (1891-1976) et par celle de Gerhard Fietz (1910-1997). À l'opposé de cette tendance, la peinture de Georg Meistermann (1911-1990) et de Josef Fassbender (1903-1974), faisant plein usage des "valeurs dramatiques ou figuratives de la forme abstraite "; enfin, Hans Hartung (1904-1989) et Wols - pseudonyme de Alfred Otto Wolfgang Schulze - (1913-1951), 
considérés comme les pionniers d'une tendance abstraite néo-expressionniste regroupant de nombreux autres artistes comme Hann Trier (1915-1999) et Bernhard Schulze (1915-2005) ${ }^{39}$.

11 Dans ce panorama, Haftmann considère Nay, Winter et Werner comme trois personnalités indépendantes. L'œuvre de ces trois artistes représente selon lui le sommet de la peinture allemande. La peinture de Nay, vidée des préoccupations concernant le contenu, est décrite par Haftmann comme le résultat d'un processus de suppression progressive de significations, d'émotions et d'éléments psychologiques, aboutissant à des taches de couleur libres qui dominent le tableau. À travers la couleur, devenue le fondement essentiel de sa peinture, Nay crée des œuvres, "gracieuses, libres et lumineuses", qui ont abandonné tout ce qui est sombre, anxieux et émotionnel, pour n'exprimer rien d'autre qu'elles-mêmes ${ }^{40}$.

12 L'insistance de Haftmann sur le concept de liberté exprimé par les œuvres d'art abstrait se relie à sa conception selon laquelle l'abstraction serait un courant artistique de prédilection pour l'homme libre du monde démocratique occidental de l'aprèsguerre, le langage artistique abstrait ne pouvant pas, à son avis, être manipulé par le pouvoir politique. Cette théorie est développée par Haftmann en réponse à l'instrumentalisation idéologique de l'art moderne par les nazis, phénomène que l'historien de l'art voyait se répéter dans le contexte de la politique culturelle mise en place par la République Démocratique d'Allemagne, qui soutenait directement le réalisme socialiste comme véhicule de messages idéologiques ${ }^{41}$. Les artistes les plus talentueux de la République Fédérale allemande d'après-guerre s'exprimeraient ainsi selon lui au moyen du langage artistique de l'abstraction. Cette opinion, Haftmann l'exprime sans détour en 1951, dans une contribution intitulée L'art abstrait à destination de la revue mensuelle des questions allemandes, L'art allemand contemporain : «Il serait faux de considérer la peinture abstraite comme représentante de l'ensemble de la recherche de la jeune peinture allemande. Mais il ne faut pas non plus ignorer que les talents les plus notoires et les plus rigoureux qui nous restent se trouvent dans le domaine des abstraits ${ }^{42} »$.

\section{7 : la mise en valeur individuelle de Nay au sein du mouvement allemand de l'abstraction}

En 1967, Haftmann écrit la préface du catalogue de l'exposition dédiée à la nouvelle production artistique de Nay, qui se tient du 14 juillet au 21 août à la Galerie Günther Franke de Munich. Dans un paragraphe intitulé Über die neuen Bilder von Nay, l'historien de l'art affirme que sur la scène de l'art contemporain les nouveaux tableaux de Nay sont une exception ${ }^{43}$. L'analyse de Haftmann montre en quoi le caractère unique de la production artistique du peintre s'oppose aux développements contemporains du Pop art et de l'art optique, auxquels l'historien de l'art nie manifestement un statut d'art et ne considère que comme des artifices et des astuces optiques. Il écrit: "Dans ce contexte éphémère, qu'une critique hâtive célèbre comme le nouveau scénario de l'expression figurative contemporaine, les nouveaux tableaux de Nay paraissent complètement isolés [...]. Cet art ne peut être rencontré sur les champs de bataille et les terrains d'ébats des artistes pop et optiques actuels, sur les aires de jeu orgiaques et divertissantes de toutes sortes de beat art, sur lesquels le monde de l'art amusé souhaite aujourd'hui s'établir. ${ }^{44}$ " 
14 La prise de position de Haftmann qui, en faisant l'éloge de Nay, exprimait des opinions très dures à l'encontre d'autres courants d'art contemporain émergeant sur la scène de l'art international, ne passa pas inaperçue et déclencha un véritable débat public dont les pages du quotidien Die Zeit se sont fait l'écho. Haftmann et Wieland Schmied (1929-2014), directeur de la Kestner-Gesellschaft de Hanovre, s'affrontèrent alors par lettres publiques interposées. Plusieurs commentaires furent également publiés, dont ceux de Nay, qui ressentait le besoin de se défendre contre ce qu'il avait interprété comme une accusation de manque de modernité ${ }^{45}$. L'historien de l'art Carl Georg Heise (1890-1979), ami et mécène de l'artiste, défendait d'une part Haftmann de l'accusation d'avoir condamné de façon arbitraire tous les nouveaux courants d'art contemporains mais, d'autre part, s'interrogeait sur la légitimité de jugement de la jeune production artistique contemporaine par des historiens de l'art appartenant à des générations différentes. Il soutenait qu'en raison de son état de gestation, l'art contemporain était un phénomène difficile à saisir dans sa complexité pour des critiques plus âgés ${ }^{46}$.

15 Dans l'une de ses lettres à Werner Haftmann, Wieland Schmied écrivait: "Cela m'attriste que l'amour sincère d'un artiste puisse ainsi amener à en considérer d'autres - selon votre texte on pourrait presque conclure: tous les plus jeunes-comme insignifiants, et à suspecter jusque leurs intentions dans leur travail, à dénier à vrai dire à des courants entiers (on pourrait penser : à tous ceux qui ont émergés au cours de la dernière décennie et demie) leur nécessité intérieure et leur engagement. L'admiration pour un artiste unique doit-elle vraiment justifier la condamnation des autres ${ }^{47}$ ?»

16 Haftmann lui fit la réponse suivante : «Le problème de la génération dans le domaine de l'art est très compliqué. [...] Qui était le peintre le plus actuel (donc dans notre terminologie le plus “jeune") en 1906 ? - C'était le vieux Cézanne! Croyez-vous, cher Monsieur Schmied, que l'on peut comprendre quelque chose au vieux contexte de la décennie 1950 à 1960 sans la puissante influence de l'œuvre tardive du vieux Matisse ou sans la - à vrai dire inattendue - grandiose portée du vieux Braque ? - Et en plus de tout cela, l'influence de l'œuvre tardive de Klee qui était décédé déjà depuis 1940 ! [...] Pour revenir au problème qui vous préoccupe, je pense donc en fait que la nouvelle production de Nay, du point de vue du développement de la couleur, est largement précurseuse - donc "plus jeune", "plus moderne", "plus actuelle" - que chacun de ces innombrables suiveurs de mode du Pop et de l'op $\operatorname{art}^{48}[. .$.$] . » En thématisant la question$ de la modernité et de l'actualité en ces termes, Haftmann revivifiait la théorie des générations élaborée par l'historien de l'art Wilhelm Pinder (1878-1947), qui proposait une vision alternative à l'idée de développement linéaire et progressif de l'art ${ }^{49}$.

\section{La double consécration du critique et de l'artiste}

17 Haftmann était conscient que son engagement pour Nay faisait l'objet de critique sur la scène artistique. Il était souvent accusé dans la presse d'avoir favorisé la carrière de son protégé qui était ainsi devenu l'artiste abstrait le plus éminent d'Allemagne ${ }^{50}$.

18 À l'occasion d'une exposition de Nay organisée à l'Akademie der Künste de Berlin Ouest du 13 janvier au 12 février 1967, Haftmann, qui vient d'être nommé directeur de la Neue Nationalgalerie, s'oppose à la demande de Nay d'inaugurer l'exposition avec un discours en sa faveur. Dans une lettre datée du 29 novembre 1966, Haftmann explique à Nay ne pas vouloir débuter son activité à Berlin par des discours publics militants dès le 
premier jour. Il ajoute avoir par ailleurs l'intention de profiter de cette exposition à l'Akademie der Künste pour compléter le fonds des œuvres de l'artiste à la Neue Nationalgalerie, qu'il considère aléatoire et insuffisant. Pourtant, il ne veut pas donner l'impression de profiter de son nouveau poste de directeur pour promouvoir exclusivement l'œuvre de son ami et lui écrit: «Puisque dans le débat au sujet de ma nomination dans les cercles artistiques berlinois émerge encore et toujours l'argument selon lequel je remplirais la Neue Nationalgalerie de tableaux de Nay, il serait très imprudent de commencer aussitôt mon activité avec un discours propagandiste pour vous (car c'est ainsi que ces cercles l'interprèteraient) et aussi de procéder immédiatement aux acquisitions dont nous avons parlé. Les deux ne passeraient pas ${ }^{51}$. "

Dans une lettre envoyée à Nay de Gmund am Tegernsee, Haftmann exprime cependant son approbation pour l'accrochage de l'exposition berlinoise et ses préférences parmi les œuvres présentées, dans la perspective d'une acquisition pour la Neue Nationalgalerie : « Votre exposition est brillante. Vous avez tout à fait raison d'appuyer tout le poids sur les dernières œuvres. [...] J'ai tout étudié à fond. Je veux les Wirbelnde Rhythmen in Blau de 1958 pour la Nat.[ional] Galerie. Y-a-t-il une chance d'acheter ce tableau? Mais surtout aussi In der Nacht de 1965 [fig. 4] qui m'impressionne beaucoup depuis déjà un certain temps. [...] S'il vous plaît, dites-moi le prix et gardez-le dans une mesure bienveillante, car je veux en avoir encore plus de vous. Entre nous, nous pouvons en discuter de manière plus approfondie [...]. Car nous avons le temps, le bâtiment de Mies ne sera prêt pour l'accrochage que l'année prochaine ${ }^{52}$.» En effet, même si dans la correspondance nous ne retrouvons plus trace de la négociation, le tableau In der Nacht a été acheté chez l'artiste en 1967 pour 24000 marks ${ }^{53}$. De plus, d'autres acquisitions pour le musée ont lieu pendant les deux premières années de travail de Haftmann. Il s'agit des tableaux Ocker, Gelb und dunkles Blau ${ }^{54}$ de 1967, donné par la Fédération de l'industrie allemande en 1968 et Der Clown zeigt sich de 1951, donné par le couple de collectionneurs et amis de Nay, Carola et Günther Peill, en $1968^{55}$. Enfin, il est fort probable que Nay aurait reçu des commandes de Haftmann pour le musée berlinois s'il n'était décédé le 8 avril 1968, quelques mois seulement avant l'inauguration en septembre $1968^{56}$. 
Figure 4.

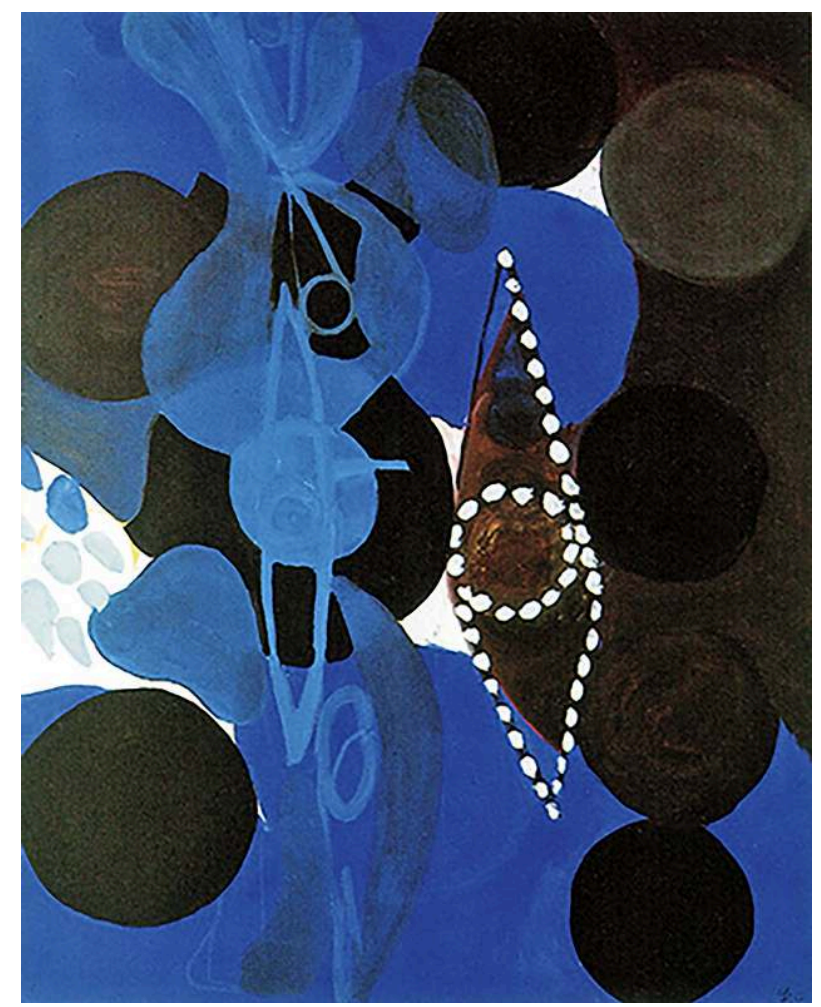

E. W. Nay, In der Nacht, 1965, huile sur toile, $200 \times 160$ cm, Berlin, Nationalgalerie, nº d' inventaire 21/67.

(c) Staatliche Museen Preußischer Kulturbesitz, Nationalgalerie

20 À l'occasion de l'enterrement de Nay à Cologne le 10 avril 1968, Haftmann affirme : «[...] désormais il ne me reste que l'obligation de donner à l'œuvre de Nay la place qu'elle mérite dans le cadre de la Neue Nationalgalerie ${ }^{57}$. » Et le 20 juin $1969^{58}$, environ un an après la mort de Nay, il inaugure une grande exposition à la Neue Nationalgalerie dédiée aux œuvres tardives de l'artiste (fig. 5), celles que Haftmann considérait comme ses chefs-d'œuvre, comme l'achèvement du langage abstrait dans sa peinture. L'accrochage compte cinquante-huit tableaux et de nombreux dessins ${ }^{59}$. Il s'agit d'une exposition itinérante organisée avec le soutien de la veuve de l'artiste, Elisabeth Nay. L'exposition circula au Wallraf-Richartz-Museum de Cologne, au Städelsches Kunstinstitut à Francfort-sur-le-Main et à la Kunstverein de Hambourg. Pour l'occasion, Haftmann rédigea la préface d'un catalogue conjoint pour les quatre expositions ${ }^{60}$. Deux aspects significatifs émergent de ce texte intitulé E. W. Nay - Das Spätwerk : l'opposition entre l'œuvre de Nay et celle des artistes de courants artistiques ayant vu le jour pendant les dernières années, opposition déjà décrite par l'historien de l'art dans le cadre de la préface au catalogue de l'exposition de Munich en 1967 ; et l'espoir qu'une génération de jeunes artistes puisse bénéficier du travail de Nay et poursuivre sur le chemin de la peinture et de l'abstraction. En effet, cette exposition avait été conçue par Haftmann comme une sorte d'avertissement pour les générations d'artistes à venir afin que, dans le monde contemporain dominé selon l'historien par la consommation et la propagande, elles puissent se souvenir de ce qu'est réellement la peinture « en tant qu'accomplissement intellectuel autonome ${ }^{61} »$. 
Figure 5 .

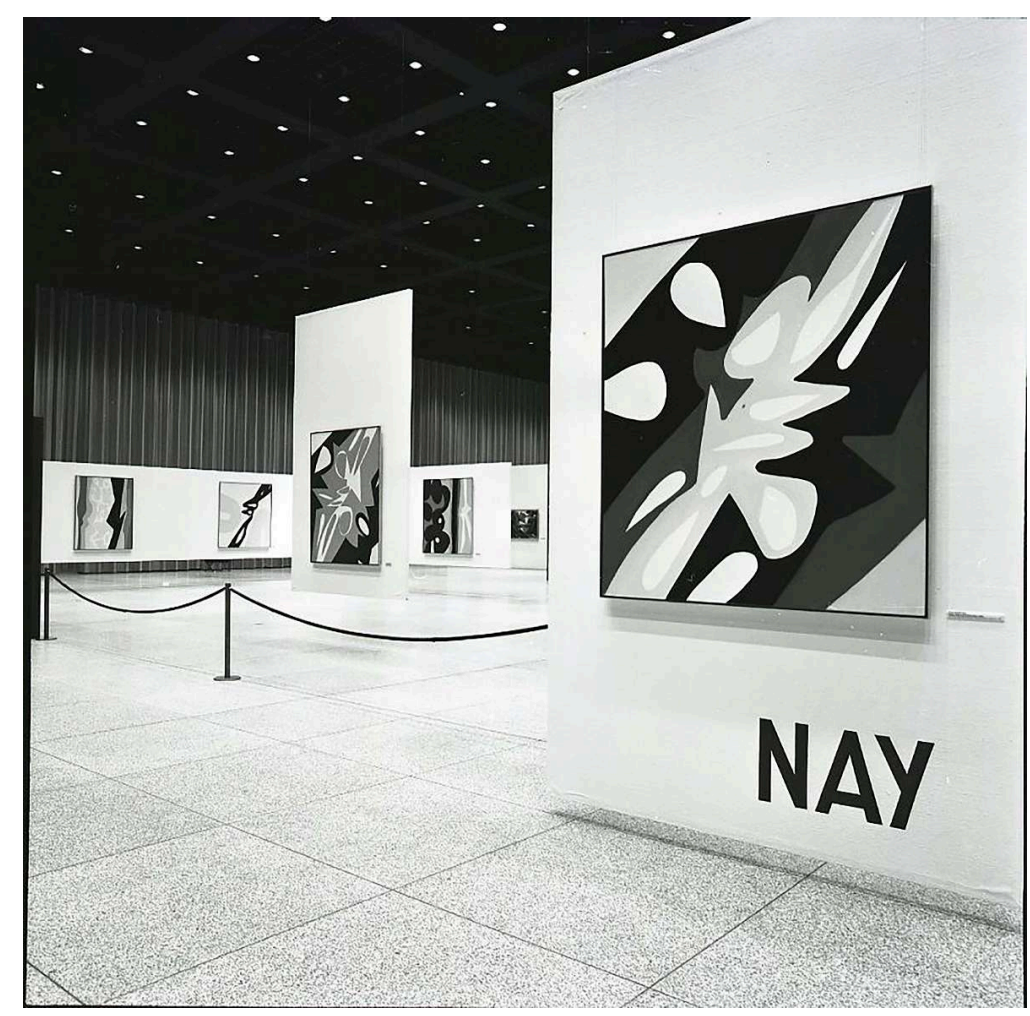

Exposition Nay, 1969, Neue Nationalgalerie, Berlin.

(c) SMB, Zentralarchiv/Reinhard Friedrich.

\section{Une relation complexe entre historien de l'art et artiste}

21 La relation entre Haftmann et Nay, qui dura de leur première rencontre à Berlin dans les années 1930 à la mort de Nay en 1968, ne s'est pas limitée au champ des relations professionnelles entre historien de l'art et artiste. À partir de l'après-guerre surtout, elle s'approfondit dans une amitié ponctuée de rencontres régulières, que ce soit à Cologne, où l'artiste habitait et travaillait, ou en Bavière, où Nay et sa femme avaient acheté une maison de vacances à proximité de celle de Haftmann, située à Gmund am Tegernsee ${ }^{62}$.

Cette relation amicale, attestée par des échanges assidus, n'a néanmoins pas été épargnée par quelques incompréhensions entre deux personnalités très différentes ${ }^{63}$. Haftmann répond ainsi le 14 décembre 1961 aux reproches de Nay, qui ne comprend pas l'engagement porté par Haftmann à des artistes très différents de lui, comme cela apparaît dans une lettre de 1961 concernant, entre autres, le peintre Georg Meistermann et ses baies vitrées crées pour les églises de Wurtzbourg et Schweinfurt ${ }^{64}$ : "Votre interprétation de mon comportement envers vous en tant que "ennemi-ami" est juste fausse. Je crois que je vous l'ai montré, puisque vous n'entendrez jamais d'aucune tierce personne honnête répéter un quelconque mot de critique à votre égard que j'aurai dit. Par ailleurs, des milliers de mots, imprimés et fixés par écrit, témoignent de mes opinions envers vous. Je crois pouvoir affirmer que pendant toutes ces années, j'ai été l'un des rares qui vous a toujours soutenu - sur le plan artistique et personnel - et vous a défendu contre toute critique. [...] Cela ne m'a pas blessé 
d'entendre à l'occasion que vous doutiez de ma loyauté ou même que vous considériez les petits troubles dans notre relation personnelle comme des doutes sur votre travail artistique. [...] Encore maintenant, je n'exige rien en retour, [...] ce que je veux dire, c'est qu'à cause de votre personnalité et de vos prédictions sur votre travail, certains aspects de mon travail et de ma curiosité vous resteront nécessairement étrangers [...]. Je suis un avocat de la mémoire, enfant de Mnémosyne, chroniqueur actif avec une vision de l'histoire et une morale à la fin..$^{65}$ "

23 Haftmann, au-delà de l'intérêt tout particulier qu'il porte à l'œuvre de Nay, se voit comme un historien voué à l'analyse des phénomènes passés de l'histoire de l'art dans le but de comprendre et ordonner les expressions du présent, et considère que garder la mémoire de démarches artistiques diverses, au travers de son travail d'écrivain et de commissaire, fait partie de sa mission, en accord avec la mère de toutes les muses, la déesse de la mémoire Mnémosyne. C'est elle qu'invoque l'historien de l'art à la fin du discours prononcé aux funérailles de Nay, en proclamant que, grâce à son travail critique, Mnémosyne aura inscrit l'œuvre de Nay dans l'éternité du souvenir, la faisant échapper à l'oubli : «Je n'ai jamais hésité à qualifier Nay d'un des plus grands peintres de notre temps et je suis fermement convaincu que les années à venir ne feront que confirmer son œuvre, car Nay a toujours eu une longueur d'avance sur nous tous. Si l'homme n'est plus là, le train de sa vie et de sa recherche a tracé une œuvre lumineuse - une constellation dans la nuit de l'histoire, une tache lumineuse devant les ténèbres de la mort qui entraîne des centaines de millions dans la nuit éternelle, mais laisse à quelques-uns leur lumière. Aux enfants de Mnémosyne, aux chanceux, au chanceux Nay. Cet éclat ne s'éteindra pas ${ }^{66}$ !»

24 Le travail de Haftmann en soutien à la peinture de Nay continua sans aucune interruption après la mort de l'artiste. Il encouragea Elisabeth Nay dans ses projets, comme en témoigne leur correspondance. Grâce à l'entremise de Haftmann ${ }^{67}$, la veuve de l'artiste rencontra ainsi, en 1969 à Cologne, Jean Leymarie, directeur du Musée National d'Art Moderne de Paris, qui avait envisagé de présenter une grande exposition rétrospective de Nay en France. Malgré l'engagement de Haftmann ${ }^{68}$ cette exposition, qui aurait été l'occasion de montrer au public français environ cinquante tableaux, quarante aquarelles et vingt dessins ${ }^{69}$, n'eut toutefois pas lieu.

\section{Épilogue d'un « combat » pour l'art abstrait}

Analysant les carrières conjointes de Haftmann et de Nay, des premières années de l'après-guerre jusqu'à la fin des années 1960, nous avons vu que ces derniers ont partagé un destin similaire. D'une part, l'historien de l'art Haftmann a essayé de délégitimer la diffusion de courants qui démentaient ses théories dogmatiques fondées sur la primauté de l'art abstrait en Occident après le Nazisme et la Seconde Guerre mondiale et son travail de critique a atteint son apogée avec sa nomination au poste de directeur de la Neue Nationalgalerie en 1967. D'autre part, l'artiste Nay a été partagé, surtout pendant les dernières années de sa vie, entre la satisfaction de la reconnaissance obtenue pour son œuvre et une préoccupation grandissante vis-à-vis du développement de tendances contemporaines qu'il considèrait peu artistiques, plutôt orientées vers le design, et menées par une nouvelle génération d'artistes en train de tourner définitivement le dos à la peinture abstraite ${ }^{70}$. Dans le contexte historique de l'après-guerre, dans un pays divisé par le rideau de fer et dominé par l'affrontement 
d'idéologies politiques opposées affectant l'histoire de l'art et la politique culturelle, nous pouvons saisir la portée du lien étroit qui a caractérisé la relation féconde entre Werner Haftmann et Ernst Wilhelm Nay - une relation fondée sur le partage d'un « combat » pour la peinture abstraite, dont la primauté est entrée en crise pendant les années 1960. On assiste alors à la naissance du Pop art, de l'art conceptuel, des arts performatifs, du Nouveau Réalisme, et ces courants, qui révolutionnent l'expression artistique en explorant de nouveaux médias et d'autres modalités pour véhiculer un message artistique. En allant au-delà de la matérialité de l'artefact ou en marquant un retour à la représentation de la réalitét ${ }^{71}$, ces nouveaux courants bouleversèrent la solidité du prétendu caractère universel de l'abstraction soutenu par Haftmann à travers les œuvres d'artistes comme Ernst Wilhelm Nay.

\section{NOTES}

1. Nous renvoyons au site internet dédié à Werner Haftmann en ce qui concerne sa bibliographie complète : www.werner-haftmann.de/literatur/ [02/05/2020]. Dans ma thèse de doctorat intitulé «Werner Haftmann - directeur de la Neue Nationalgalerie à Berlin. Expositions temporaires et politique d'acquisition (1967-1974) ", à soutenir en 2021, co-encadrée à l'Université de Heidelberg et à l'École du Louvre de Paris sous la direction de Monsieur Henry Keazor et Madame Cecilia Hurley-Griener, j'analyse en détail la biographie de l'historien de l'art, en soulignant les omissions et les inexactitudes de cette biographie publiée par la veuve de l'historien de l'art, Evelyn Haftmann. Le projet de recherche est soutenu par une bourse du Land BadenWürttemberg au Zentralinstitut für Kunstgeschichte à Munich (2017-2019), une bourse Paris X Rome au Centre allemand d'histoire de l'art, Max-Weber-Stiftung de Paris et à la Bibliotheca Hertziana, Max-Planck-Institut für Kunstgeschichte de Rome (2019-2020), une bourse pour l'achèvement de la thèse de la part de la Landesgraduiertenförderung der Graduiertenakademie de l'Université de Heidelberg (2020) et une bourse en soutien de la cotutelle de thèse de la part de l'Université franco-allemande (2020-2021).

2. Nous renvoyons au site internet de la Ernst Wilhelm Nay Stiftung pour une biographie détaillée de l'artiste : www.ewnay.de/der-kuenstler/ [02/05/2020].

3. Cet article est issu d'une présentation donnée à l'occasion de la journée d'étude «Ernst Wilhelm Nay et l'abstraction d'après-guerre ", organisée par Barbara Honrath (Goethe-Institut, Paris), Thomas Kirchner (DFK, Paris), Angela Lampe (Centre Pompidou, Paris) avec le soutien de la Ernst Wilhelm Nay Stiftung, Cologne,qui a eu lieu le 25 mars 2019 au Centre allemand d'histoire de l'art à Paris.

4. Haftmann s'est dédié à l'étude et à la promotion de l'œuvre de Nay à partir des premières années de l'après-guerre. Il a écrit de nombreux articles, préfaces de catalogues, discours d'inauguration pour les expositions, ainsi que l'ouvrage Ernst Wilhelm Nay, publié à Cologne par la maison d'édition DuMont Schauberg en 1960 et réédité en 1991.

5. La rencontre entre Haftmann et Nay date de l'avant-guerre à Berlin, comme cela a été confirmé par le galeriste Aurel Scheibler, fils de la deuxième femme de l'artiste, Elisabeth Nay, et gérant de l'héritage de Nay lors de la journée d'étude au Centre allemand d'histoire de l'art à Paris La mention la plus ancienne concernant Nay que nous avons retrouvée dans la correspondance de Haftmann se trouve dans une lettre datée du 16 janvier 1942, adressée à Hans 
Purrmann, directeur de la Villa Romana de Florence et envoyée de Turin de la part de Haftmann. Haftmann y évoquait le fait que Nay avait exposé à la Galerie Günther Franke de Munich. Correspondence avec Werner Haftmann, Hans Purrmann Archiv, Munich.

6. Nous renvoyons à ce propos à la publication suivante, qui traite, entre autres, du rôle joué par Haftmann dans l'accrochage des œuvres de Nay à la Documenta III en 1964 : Dirk Schwarze, Die Kunst der Inszenierung oder als Arnold Bode Ernst Wilhelm Nay in den Himmel hob, Berlin, B \& S Siebenhaar Verlag, 2009.

7. Werner Haftmann, Malerei im 20. Jahrhundert, Munich, Prestel Verlag, 1954. Cette publication, suivie en 1955 par un volume illustré, est considérée comme un ouvrage fondamental pour l'étude de l'art du XXe siècle. Elle a été rééditée maintes fois en Allemagne (de 1957 à 2000) et diffusée aussi à l'étranger au moyen de traductions en anglais et en italien publiées en 1960. Depuis l'édition modifiée et élargie de 1962, intitulée Malerei im 20. Jahrhundert. Eine Entwicklungsgeschichte, l'encyclopédie inclut également l'art des États-Unis et de la GrandeBretagne. Notre étude est basée sur la réédition de 1987 de cette édition.

8. W. Haftmann, op. cit. note 7, p. 503.

9. Idem, ibidem.

10. Idem, ibidem, pp. 501-503.

11. «Malen, das heißt aus der Farbe das Bild formen. » W. Haftmann, op. cit. note 7, p. 503.

12. Idem, ibidem, p. 504.

13. Idem, ibidem, pp. 504-505.

14. Dirk Schwarze, « Die Karriere einer Ausstellung 60 Jahre documenta », Blickpunkt Hessen, édité par le Hessische Landeszentrale für politische Bildung, Nr. 19, Wiesbaden, 2015, p. 3.

15. Cat. d'exp. Documenta. Kunst des XX. Jahrhunderts. Internationale Ausstellung, sous la direction d'Arnold Bode, Museum Fridericianum, Kassel, 15 juillet-18 septembre 1955, Munich, Prestel Verlag, 1955.

16. D. Schwarze, op. cit. note 14, p. 4.

17. Idem, ibidem, p. 5.

18. Idem, ibidem, p. 6.

19. Werner Haftmann, « Von den Inhalten der modernen Kunst, Rede anläßlich der Eröffnung der „documenta II“ am 11.05.1959 », Das antwortende Gegenbild. Ausgewählte Texte 1947-1990, édité par Evelyn Haftmann et Wouter Wirth, Hirmer Verlag, Munich, 2012, pp. 53-55.

20. Harald Kimpel, Karin Stengel, Documenta II. Kunst nach 1945. Internationale Ausstellung. Eine fotografische Rekonstruktion, Brême, Ed. Temmen, 2000, p. 9.

21. Idem, ibidem.

22. Idem, ibidem, pp. 42-43.

23. Werner Haftmann, « Die institutionelle Gefahr, Rede anläßlich der Eröffnung der „documenta III“ am 27.6.1964», op. cit. note 19, pp. 59-68.

24. «Ce qui compte dans cette situation c'est uniquement l'esprit créateur individuel [...]. Sans aucune intention préconçue de connexion, sans égard au style et à l'argumentation nous avons placé individualité à côté d'individualité et nous avons fait attention seulement à écouter la voix individuelle, qui a réveillé en nous un écho ou qui nous a promis une découverte poétique. »Idem, ibidem, p. 67.

25. D. Schwarze, op. cit. note 14, p. 9.

26. D. Schwarze, op. cit. note 6, pp. 18-23.

27. Idem, ibidem, pp. 49-51.

28. Harald Kimpel, Karin Stengel, Documenta III. Internationale Ausstellung. Eine fotografische Rekonstruktion, Brême, Ed. Temmen, 2005, pp. 5-11.

29. Camilla Blechen, Als die Maler hören lernten. Haftmanns Abschied: Die Ausstellung „Hommage à Schönberg" in der Neuen Nationalgalerie Berlin / Wechselwirkungen moderner Musik und Malerei, Frankfurter Allgemeine Zeitung, 3 octobre 1974. 
30. Jörn Merkert, Nachruf auf Prof. Dr. Werner Haftmann anläßlich der 13. Mitgliederversammlung der Akademie der Künste Berlin-Brandenburg, am Sonnabend, dem 9. November 1999, AdK, Berlin, 0785 : Abt. Bildende Kunst Nachrufe 1993-2002.

31. Dans la presse allemande Haftmann était souvent accusé de manquer d'impartialité et de privilégier les courants non figuratifs comme l'art informel et l'abstraction. Voir par exemple l'article d'Heinz Ohff, «Fünfzehn Jahre zu spät. Werner Haftmann Direktor der NationalGalerie? ", Der Tagespiegel, Berlin, 6 mai 1966, dans lequel le critique d'art soutenait entre autres que c'était grâce à l'aide de Haftmann que Nay était devenu le plus éminent artiste abstrait en Allemagne.

32. Alfred Hentzen (1903-1985), à l'époque directeur de la Kunsthalle de Hambourg, est chargé de la section dédiée à la sculpture et William S. Lieberman (1924-2005), commissaire des arts graphiques au MoMA, s'occupe de la préface relative aux arts graphiques. Cat. d'exp., German art of the twentieth century, sous la direction de Andrew Carnduff Ritchie, MoMA, New York, 2 octobre- $^{\text {er }}$ décembre 2017, Berlin, Brüder Hartmann, 1957.

33. Dorothea Schöne estime que le but envisagé de proposer une vue d'ensemble complète de l'art allemand $\mathrm{du} \mathrm{XX}{ }^{\mathrm{e}}$ siècle n'a pas été atteint et considère que l'accent a finalement été mis sur l'art de l'avant-guerre. Dorothea Schöne, Freie Künstler in einer freien Stadt. Die amerikanische Förderung der Berliner Nachkriegsmoderne, Berlin, De Gruyter, 2016, p. 181.

34. A. C. Ritchie, op. cit. note 32, p. 11.

35. Kurt Martin joue un rôle déterminant en ce qui concerne le choix des artistes et des œuvres à présenter dans le cadre de l'exposition. D. Schöne, op. cit. note 33, p. 184.

36. Carte de la part de Werner Haftmann à Kurt Martin datée du 22 mars 1957, Germanisches Nationalmuseum, Deutsches Kunstarchiv und Historisches Archiv, Nuremberg, DKA NL Martin, Kurt, 177.

37. W. Haftmann, op. cit. note 32, p. 130.

38. Idem, ibidem, p. 135.

39. Idem, ibidem, pp. 132-136.

40. Idem, ibidem, p. 139.

41. W. Haftmann, op. cit. note 19, pp.41-58; Werner Haftmann, «Malerei nach 1945. Einführung ", cat. d'exp. Documenta II., Kunst nach 1945. Internationale Ausstellung, sous la direction d'Arnold Bode, Museum Fridericianum, Kassel, 11 juillet-11 octobre 1959, DuMont Schauberg, Cologne, 1959, pp. 11-19.

42. Werner Haftmann, «L'art abstrait », L'art allemand contemporain, Documents, Revue mensuelle des questions allemandes, Numéro spécial édité par le Bureau International de Liaison et de Documentation Offenbourg en Bade 1951, p. 70.

43. Werner Haftmann, «Über die neuen Bilder von Nay », cat. d'exp., Galerie Günther Franke, Munich, 1967, pas numéroté.

44. "In diesem ephemeren Kontext, den eine eilfertige Kritik als den neuen Entwurf zeitgenössischen bildnerischen Ausdrucks feiert, erscheinen die neuen Bilder Nays ganz isoliert [...]. Auf den heutigen Kampf- und Tummelplätzen der Pop- und Op-Artisten also, auf den orgiastischen und vergnüglichen Spielplätzen von allerlei Beat-Kunst, auf denen sich die amüsierte Kunstwelt heute einzurichten wünscht, läßt sich dieser Kunst nicht begegnen. » Idem, ibidem. En traduisant toutes les citations d'ouvrages et de lettres contenues dans cet article de l'allemand au français, nous avons respecté le style de Haftmann.

45. Lettre de la part d'Ernst Wilhelm Nay, $1^{\text {er }}$ septembre 1967, Die Zeit : www.zeit.de/1967/35/ monomanie-des-kuenstlers [2 mai 2020].

46. Lettre de la part de Carl Georg Heise, 8 septembre1967, Die Zeit: www.zeit.de/1967/36/ kampf-der-dioskuren [2 mai 2020]. Heise avait exprimé ce point de vue dans le catalogue de l'exposition au Museum Folkwang à Essen en 1962. Cat. d'exp., E. W. Nay 60 Jahre, sous la direction 
de Heinz Köhn, Museum Folkwang, Essen, 15 septembre-21 octobre 1962, Cologne, DuMont Schauberg, 1962, p. 16.

47. « Mich betrübt, wie die aufrichtige Liebe zu einem Künstler dazu führen kann, andere - fast könnte man aus Ihrem Text schließen : alle jüngeren - gering zu achten und mit ihrem Werk auch ihre Absichten zu verdächtigen, ja ganzen Richtungen (man könnte meinen : allen in den letzten anderthalb Jahrzehnten hervorgetretenen) die innere Notwendigkeit und Verbindlichkeit abzusprechen. Muß denn wirklich die Verehrung des einen Künstlers immer gleich das Argument der Verurteilung des anderen abgeben? » Lettre de la part de Wieland Schmied à Werner Haftmann, 18 août 1967, Die Zeit : www.zeit.de/1967/33/ihr-urteil-betruebt-mich [2 mai 2020].

48. « Das Problem der Generation liegt in der Kunst ungemein kompliziert. [...] Wer war 1906 der „aktuellste“ (in unserer Terminologie also der ,jüngste“) Maler? - Es war der alte Cézanne! Glauben Sie, lieber Herr Schmied, daß etwa der alte Kontext des Jahrzehnts zwischen 1950 und 1960 begriffen werden kann ohne den mächtigen Einschuß des Spätwerkes des alten Matisse oder ohne den - eigentlich unerwarteten - grandiosen Wurf des alten Braque ? - Und dazu die Wirkung des späten Klee, der schon 1940 gestorben war ! [...] Um auf das Sie beschäftigende Problem zurückzukommen, so glaube ich in der Tat, daß der neue Abschnitt im Werk E. W. Nays von der Entwicklung der Koloristik her gesehen weitaus zukunftsweisender - also „jünger“, „moderner“, ,aktueller“ - ist als jene zahllosen mitlaufenden Spielfolgen von Pop und op [...]. » Lettre de la part de Werner Haftmann à Wieland Schmied, 15 septembre 1967, Die Zeit: www.zeit.de/1967/37/nicht-nur-junge-kuenstler-schaffen-junge-kunst [02/05/2020].

49. Voir à ce propos : Sabine Fastert, «Pluralismus statt Einheit. Die Rezeption von Wilhelm Pinders Generationenmodell nach 1945 », Kunstgeschichte nach 1945. Kontinuität und Neubeginn in Deutschland, dirigé par Nikola Doll, Ruth Heftrig, Olaf Peters, Ulrich Rehm, Cologne, Böhlau, 2006, pp. 51-65. L'utilisation des théories de Pinder met en évidence l'influence problématique, dans les écrits de Haftmann, du travail de l'un des historiens de l'art nazis les plus influents. Cet aspect, ainsi que la relation ambivalente de Haftmann avec le régime nazi, qui ne peuvent être analysé dans cet article, sont traités en profondeur dans ma thèse de doctorat.

50. H. Ohff, op. cit. note 31.

51. «Da in der unterschwelligen Diskussion über meine Berufung in Berliner Künstlerkreisen immer wieder das Argument auftaucht, ich würde ja doch mir die Nat.[ional] Galerie mit NayBildern vollstopfen, wäre es sehr unklug, meine Tätigkeit sogleich mit einer Propagandarede für Sie zu beginnen, (denn so würden es diese Kreise auslegen) und auch sogleich mit entsprechenden Ankäufen zu beginnen. Beides geht nicht. » Lettre de la part de Werner Haftmann à E. W. Nay datée du 29 novembre 1966, Germanisches Nationalmuseum, Deutsches Kunstarchiv und Historisches Archiv, Nuremberg, DKA, Nay E. W. M I, C-97.

52. "Ihre Ausstellung ist glänzend. Vollkommen richtig, dass Sie das ganze Gewicht auf die später Bilder legten. [...] Ich habe alles gründlich studiert. Für die Nat.[ional] Galerie wolle ich mir aus die „Wirbelnden Rhythmen in Blau“ von 1958. Bestünde da eine Aussicht, das Bild kaufen zu können? Besonders aber auch „In der Nacht“ von 1965, was mir schon seit einiger Zeit sehr imponiert. [...] Bitte, nennen Sie mir doch den Preis und halten sie diesen in wohlvollender Rahmen, denn ich werde noch mehr von Ihnen haben wollen. Darunter können wir uns noch gründlich beraten [...]. Denn wir haben Zeit, der Mies-Bau wird erst im nächsten Jahr hängefertig. » Lettre de la part de Werner Haftmann à E. W. Nay non datée (certainement datée du début de 1967, car l'exposition de Nay à l'Académie der Künste a eu lieu du 13 janvier au 12 février 1967), Germanisches Nationalmuseum, Deutsches Kunstarchiv und Historisches Archiv, Nuremberg, DKA, Nay E. W. M I-C, 97.

53. Liste des acquisitions, SMB-ZA, II B/NG 037, VA6792, Schriftwechsel und Angebote von Privatpersonen, Kunsthallen und Galerien A-J, 1967-1975. 
54. Cette œuvre fut donnée à l'occasion de l'inauguration du musée et appartient aux derniers tableaux peints par l'artiste. Haftmann décrit ainsi le tableau: «Ces derniers tableaux de Nay représentent la somme de toutes les idées précédentes du grand coloriste qu'était Nay. [...] Si l'on cherchait une similitude, alors l'on pourrait comparer cela seulement avec le dernier Matisse, le Matisse des énormes "Papiers coupés". [...] L'image récemment acquise marque le grand point final dans l'œuvre de Nay. » Werner Haftmann, « Neuerwerbungen der Nationalgalerie in 1968 », Jahrbuch Preußischer Kulturbesitz, vol. 6, Berlin, Gebr. Mann Verlag, 1968, pp. 200-201.

55. Carte de la part d'Alexander Drückers à la Gazette des Beaux-Arts, Paris, datée du 11 novembre 1968, SMB-ZA, VA11162, Schriftwechsel A-L, 1968, Bandnummer 01, Lettre G.

56. «J'ai vu Nay en dernier lieu à Berlin. Nous avons regardé le nouveau bâtiment de mon musée et j'ai tellement espéré qu'il inspirerait Nay pour la création d'un tableau grand et radieux. » Werner Haftmann, Totenrede für Nay, Germanisches Nationalmuseum, Deutsches Kunstarchiv und Historisches Archiv, Nuremberg, DKA NL, Martin, Kurt, 855.

57. «[...] mir bleibt nur die Verpflichtung, dem Werk Nays im Rahmen der Nationalgalerie den Rang zu geben, der ihm gebührt. » Idem, ibidem.

58. Carte d'invitation à l'inauguration de l'exposition «E. W. Nay (1902-1968) Gemälde der letzten Jahre» le vendredi 20 juin 1969 à 20 heures, SMB-ZA, VA10042, Einladungen und Pressemitteilungen zu Ausstellungen NNG.

59. Cat. d'exp., E. W. Nay (1902 - 1968), sous la direction de Werner Haftmann, Wallraf-Richartz Museum, Cologne, 18 avril-8 juin 1969, Neue Nationalgalerie, Berlin, 21 juin-28 juillet 1969, au Städelsches Kunstinstitut, Francfort-sur-le-Main, 8 août-21 septembre 1969, Kunstverein, Hambourg, 4 octobre-16 novembre 1969, Müller, Cologne 1969.

60. Carte de la part de Werner Haftmann à la Direction générale des musées nationaux datée de 29 mai 1969, SMB-ZA, VA10150, Ausstellung Ernst Wilhelm Nay, 20.06.-28.07.1969.

61. W. Haftmann, op. cit. note 59 , p. 8.

62. Carte de la part de Werner Haftmann à Madame Neumeyer datée de 16 février 1967 : «J'ai vu Nay très souvent ces derniers temps. Il a acheté il y a environ deux ans une jolie maison de vacances près de Tegernsee [...]. » Germanisches Nationalmuseum, Deutsches Kunstarchiv und Historisches Archiv, Nuremberg, DKA NL Neumeyer, Alfred IC 48, Haftmann, Werner Kh.

63. Haftmann se réfère à la personnalité «anguleuse » de Nay dans une lettre adressée à $\mathrm{E}$. W. Nay datée de 14 décembre 1961, Germanisches Nationalmuseum, Deutsches Kunstarchiv und Historisches Archiv, Nuremberg, DKA, Nay, E. W. M I, C-97. De plus, Haftmann écrit à Erhard Göpel le 20 février 1958: «J'ai vu Nay à Cologne [...] Cette personne a tellement besoin de friction! Mais il est aussi trop seul. La friction est sa façon de garder le contact social et humain. [...] Après tout, c'est un peintre merveilleux, le plus peintre de tous. » Ana 415, Haftmann, Werner an Göpel, Bayerische Staatsbibliothek, Abteilung Handschriften, Munich.

64. Il s'agit d'une lettre de la part de Werner Haftmann à E. W. Nay datée "Dimanche, soir » : «Vous savez que je ne partage pas votre opinion sur Meistermann en tant que personne. [...] Vous ne devez pas oublier que je suis un historien, qui par curiosité pour le présent et son existence, essaie de connaître le contemporain à travers de la plus grande documentation possible. [...] Je m'intéresse donc à la mosaïque du contemporain [...]. C'est pourquoi je m'occupe d'artistes que vous (et d'autres) considérez une abomination. » Germanisches Nationalmuseum, Deutsches Kunstarchiv und Historisches Archiv, Nuremberg, DKA, Nay, E. W. M I, C-97.

65. «Ihre Deutung meines Verhältnisses zu Ihnen als „Feind-Freundschaft“ ist nur [?] falsch. Ich glaube, das auch gezeigt zu haben, denn Sie werden nie von irgendeinem ehrlichen Dritten irgendein Wort der Kritik Ihnen gegenüber gehört haben, das ich von mir gegeben hätte. Im übrigen stehen viele Tausende von Worte - gedruckt und fixiert - als Zeugnis meine Verhältnisses zu Ihnen. Ich glaube sagen zu können, dass ich in diesen vielen Jahren einer der weniger gewesen bin, der Ihnen unentwegt - und zwar künstlerisch wie menschlich - die Stange gehalten hat und Sie gegen jede Kritik verteidigt hat. [...] Es hat mich auch nie verletzt, wenn ich 
gelegentlich hören musste, dass Sie an meiner Loyalität zweifelten oder ja kleine Trübungen in unserem persönlichen Verhältnis bereit als Zweifel an Ihrer Arbeit als Künstler ansahen. Ich verlange auch jetzt [...] keine Gegenleistung, mein [sic] ich doch, dass, aus der Struktur Ihrer Persönlichkeit und Ihren Vorausschaungen zu Ihrer Arbeit, bestimmte Seiten in der Richtung meiner Arbeit und meiner Neugierde Ihnen notwendig fremd bleiben müssen [...]. Ich bin Verwalter der Erinnerung, Kind der Mnemosyne, aktiver Chronist mit einer Geschichtsvision und einer Moral am Ende. " Lettre de la part de Werner Haftmann à E. W. Nay datée de 14 décembre 1961, Germanisches Nationalmuseum, Deutsches Kunstarchiv und Historisches Archiv, Nuremberg, DKA, Nay, E. W. M I, C-97.

66. «Ich habe nie gezögert, Nay einen der größten Maler unserer Zeit zu nennen, und ich bin fest überzeugt, daß die kommenden Jahre sein Werk erst bestätigen werden, - denn Nay war uns allen immer einen Schritt in die Zukunft voraus. Ist auch der Mann nicht mehr, so ist doch die Bahn seines Lebens und seines Suchens in einem leuchtenden Werk aufgeschrieben, - ein Sternbild in der Nacht der Geschichte, ein Leuchtpunkt vor dem Dunkel des Todes, der Hunderte von Millionen in die ewige Nacht reißt, aber einigen Wenigen ihr Leuchten läßt : - den Kindern der Mmemosyne, den Glückskindern, dem Glückskind Nay. Dieses Leuchten wird nicht aufhören. » W. Haftmann, op. cit. note 56.

67. Carte de la part de Werner Haftmann à Jean Leymarie datée du 3 décembre 1968 : «Ces joursci j'ai recu [sic] une lettre de Elisabeth Nay, la femme du peintre E. W. Nay, un de mes plus chers amis décédé il y a plusieurs mois. Tu connais certainement quelques travaux de lui ; Jean Cassou connaissait Nay personellement [sic] et peut t'en parler. Elisabeth Nay est actuellement en train de préparer une exposition commémorative d'environ 70 toiles que je montrerai à la Nationalgalerie à Berlin. Elle est également très désireuse que cette exposition soit présentée à Paris [...]. Je te prie de me faire connaitre [sic] ton opinion sur E. W. Nay comme artiste et si cette exposition t'intéresse pour ton musée." SMB-ZA, VA11162, Schriftwechsel A-L, 1968, Bandnummer 01, L.

68. Sous demande d'Elisabeth Nay (Carte de la part d'Elisabeth Nay à Werner Haftmann datée du 25 Mai 1969, SMB-ZA, VA10150, Ausstellung Ernst Wilhelm Nay, 20.06.-28.07.1969.), Haftmann invite Jean Leymarie à l'inauguration de l'exposition à la Neue Nationalgalerie en lui remboursant les frais de voyage. Lettre de la part d'Haftmann à Jean Leymarie datée du 4 juin 1969, SMB-ZA, VA10150, Ausstellung Ernst Wilhelm Nay, 20.06.-28.07.1969.

69. Carte de la part d'Elisabeth Nay à Werner Haftmann datée du 25 mai 1969, SMB-ZA, VA10150, Ausstellung Ernst Wilhelm Nay, 20.06.-28.07.1969.

70. W. Haftmann, op. cit. note 59, p. 7.

71. Voir à ce propos l'introduction au catalogue de la Documenta III, dans lequel Haftmann se positionne contre les collectifs d'artistes ainsi que contre le pop art. Werner Haftmann, «Einführung documenta III », cat. d'exp., Documenta III. Internationale Ausstellung, Alte Galerie, Museum Fridericianum, Kassel, 28 juin-6 octobre 1964, édité par Siegfried Hagen et Alfred Nemeczek, Cologne, DuMont Schauberg, 1964, pp. 14-17.

\section{RÉSUMÉS}

La relation professionnelle et amicale entre l'historien de l'art Werner Haftmann (1912-1999) et l'artiste abstrait Ernst Wilhelm Nay (1902-1969) dans la République fédérale d'Allemagne pendant l'apres-guerre répresente un cas emblématique d'élaboration d'une historiographie de l'art. 
À travers l'analyse de la correspondance, de l'encyclopédie de l'art moderne Malerei im 20. Jahrhundert, publiée par Haftmann en 1954, et des catalogues d'expositions, cette étude retrace les étapes fondamentales du parcours de consécration de la peinture abstraite de Nay entrepris par l'historien de l'art en Allemagne et à l'international. D'un côté, Nay a profité considérablement du soutien critique de la part d'un des historiens de l'art les plus influents de l'Allemagne de l'Ouest. De l'autre, Haftmann a forgé autour de l'œuvre et de la figure du peintre la démonstration exemplaire de ses théories proclamant le triomphe de l'art abstrait respectivement comme art du futur monde démocratique occidental de l'après-guerre ainsi que prestation créatrice libre et indépendante.

The professional and amicable relationship between the art historian Werner Haftmann (1912-1999) and the abstract artist Ernst Wilhelm Nay (1902-1969) in the Federal Republic of Germany during the post-war period is an emblematic case of the development of an art historiography. Through the analysis of correspondence, the modern art encyclopedia Malerei im 20. Jahrhundert, published by Haftmann in 1954, and exhibitions catalogues, this study retraces the fundamental stages of the path to the consecration of Nay's abstract painting undertaken by the art historian in Germany and internationally. On the one hand, Nay benefited considerably from the critical support of one of West Germany's most influential art historians. On the other hand, Haftmann forged around the work and the figure of the painter the exemplary demonstration of his theories proclaiming the triumph of abstract art respectively as the art of the future western democratic post-war world as well as a free and independent creative performance.

\section{INDEX}

Index chronologique : après-guerre

Keywords : Ernst Wilhelm Nay, Werner Haftmann, Germany, abstract art, post-war, documenta, Neue Nationalgalerie, correspondence, archives

Mots-clés : Ernst Wilhelm Nay, Werner Haftmann, Allemagne, art abstrait, documenta, Neue Nationalgalerie, correspondance, archives

\section{AUTEUR}

\section{VINCENZA BENEDETTINO}

Vincenza Benedettino est doctorante en cotutelle à l'Universität Heidelberg et à l'École du Louvre avec la thèse intitulée Werner Haftmann directeur de la Neue Nationalgalerie à Berlin. Expositions temporaires et politique d'acquisition (1967-1974). Elle a été boursière du Land Baden-Württemberg au Zentralinstitut für Kunstgeschichte à Munich de 2017 à 2019 et boursière « Paris x Rome » au Centre allemand d'histoire de l'art, Max Weber Stiftung à Paris et à la Bibliotheca Hertziana, Max Planck Institut für Kunstgeschichte à Rome de 2019 à 2020. Auparavant elle a étudié histoire de l'art et muséologie à l'Università degli Studi di Trieste, à l'Universität Heidelberg, à l'École du Louvre et à l'Université de Montréal dans le cadre du séminaire de muséologie canadienne en coopération avec l'École du Louvre.

Vincenza Benedettino is a doctoral student at both the Universität Heidelberg and the École du Louvre with a thesis entitled Werner Haftmann directeur de la Neue Nationalgalerie à Berlin. Expositions temporaires et politique d'acquisition (1967-1974). She held a scholarship from the Land Baden-Württemberg au Zentralinstitut für Kunstgeschichte in Munich from 2017 to 2019, held 
the "Paris x Rome" scholarship from the Centre Allemand d'Histoire de l'Art, Max Weber Stiftung in Paris, and at the Bibliotheca Hertziana, Max Planck Institut für Kunstgeschichte in Rome from 2019 to 2020. She previously studied art history and museology at the Università degli Studi di Trieste, at the Universität Heidelberg, at the École du Louvre and at the Université de Montréal as part of the Canadian museology seminar in cooperation with the École du Louvre. 